\title{
Association Between Maternal Glucose/Lipid Metabolism Parameters and Abnormal Newborn Birth Weight in Gestational Diabetes Complicated by Preeclampsia: A Retrospective Analysis of 248 Cases
}

\author{
Yunshan Xiao (D) Xueqin Zhang
}

Received: December 30, 2019 / Published online: February 27, 2020

(C) The Author(s) 2020

\begin{abstract}
Introduction: Women with gestational diabetes mellitus (GDM) with co-existent preeclampsia (GCP) are at increased risk of giving birth to a baby with an abnormal birth weight. We have analyzed the risk factors for abnormal newborn birth weight (NBW) in women with co-presence of GDM and GCP, focusing on maternal glucose/lipid metabolism, with the aim to optimize the clinical intervention strategy.

Methods: The clinical data of 248 pregnant women with GCP and their infants were retrospectively analyzed through a comprehensive review of the electronic medical records of Women and Children's Hospital, Xiamen University (Xiamen, China). These women had received prenatal care and had their baby delivered in the hospital between January 2016 and November 2018. Major characteristics
\end{abstract}

Yunshan Xiao and Xueqin Zhang contributed equally to this work and should be considered co-first authors.

Enhanced Digital Features To view enhanced digital features for this article go to https://doi.org/10.6084/ m9.figshare.11854605.

Y. Xiao $(\bowtie) \cdot$ X. Zhang

Department of Obstetrics, Women and Children's

Hospital, Xiamen University, Xiamen 361003,

Fujian, People's Republic of China

e-mail: xyssfp@163.com assessed were large for gestational age (LGA), small for gestational age (SGA), severe preeclampsia (S-PE), and maternal plasma glucose/lipid profile in late pregnancy. Secondary characteristics were maternal age, height, body mass index (BMI), gestational weight gain (GWG), abortion history, education level, primipara or not, preterm or not, and fetal gender. Regression analysis was used to analyze the association between maternal glucose/lipid metabolism parameters and LGA or SGA.

Results: There was no difference in the ratio of advanced maternal age, primipara, abortion history, preterm delivery, and newborn sex between the control group and the LGA or SGA group. Logistic regression analysis, with such factors as maternal stature, BMI, among others, was applied. Multivariate analysis of SGA infants revealed the following associations: S-PE (odds ratio [OR] 3.226, 95\% confidence interval [CI] 1.385-7.515; adjusted OR [AOR] 3.675, 95\% CI $1.467-9.207 ; p<0.05)$; high levels of glycated hemoglobin (HbA1c > 6.5\%) (OR 0.436, 95\% CI 0.187-1.017; AOR 0.459, 95\% CI 0.179-1.173; $p$ $>0.05$ ); low levels of high-density lipoprotein cholesterol (HDL-C $<1.0 \mathrm{mmol} / \mathrm{L}$ ) (OR 0.625, 95\% CI 0.287-1.361; AOR 0.637, 95\% CI $0.267-1.520 ; p>0.05)$. Multivariate analysis of LGA revealed the following associations: S-PE (OR 30.885, 95\% CI 0.398-2.013; AOR 0.974, 95\% CI $0.400-2.371 ; p>0.05)$; high levels of HbA1c (OR 4.542, 95\% CI 0.187-11.824; AOR 3.997, 95\% CI 1.452-10.998; $p<0.05)$; low 
levels of HDL (OR 3.393, 95\% CI 1.362-8.453; AOR 2.900, 95\% CI 1.100-7.647; $p<0.05$ ).

Conclusions: The results of our analysis revealed that severity of preeclampsia was associated with SGA. The high HbA1c and low HDL-C values found in our analysis were independent risk factors for LGA in women with GCP, while other lipoproteins were not associated with abnormal NBW. These findings suggest that there are differences in the effects of various maternal lipid parameters on NBW.

Keywords: Gestational diabetes mellitus; Glucose/lipid metabolism; HbA1c; HDL-C; Preeclampsia

\section{Key Summary Points}

\section{Why carry out this study?}

It is essential to identify the risk factors for abnormal newborn birth weight (NBW) to improve the clinical management of gestational diabetes mellitus complicated by preeclampsia (GCP). Previous studies on GCP have tended to be undermotivated.

The purpose of this study was to investigate the association between maternal glycolipid metabolism parameters and the abnormal NBW in women with GCP.

\section{What was learned from this study?}

The severity of preeclampsia was found to be associated with small for gestational age newborns. High glycated hemoglobin A1c and low high-density lipoprotein cholesterol values were independent risk factors for large for gestational age infants in women with GCP, while other lipoproteins were not associated with abnormal NBW.

The study highlights the importance of carrying out timely risk monitoring and identifying the early warning signs, with the aim to improve infant health.

\section{INTRODUCTION}

Newborn birth weight (NBW), which refers to the weight of a newborn measured within $1 \mathrm{~h}$ after birth, is a good indicator of intrauterine fetal development and maternal nutrition. There are two types of NBW abnormalities according to the gestational age and sex-specific INTERGROWTH-21st curves: small for gestational age (SGA; birth weight $<10$ th percentile) and large for gestational age (LGA; birth weight $>$ 90th percentile) [1]. SGA is associated with increased newborn morbidity and mortality and an increased risk of developmental dysfunction and metabolic disease in later life [2]. LGA may lead to fetal brachial plexus injury, intracranial hemorrhage, and shoulder dystocia during delivery. It is also associated with long-term complications, such as various metabolic diseases and cardiovascular diseases [3].

NBW is closely related to the health status of the mother during pregnancy. It has been reported that women with gestational diabetes mellitus (GDM) or preeclampsia have an increased risk of an abnormal NBW. GDM is defined as hyperglycemia that is recognized for the first time during pregnancy, excluding cases of overt diabetes prior to gestation. The main manifestation of preeclampsia is apparent hypertension after 20 weeks of pregnancy, which may be accompanied by proteinuria, edema, and other symptoms [4]. In recent years, the incidence of GDM has increased gradually, reaching $17.5 \%$ in China [5]. Sun et al. reported that the incidence of preeclampsia in GDM patients was $8.7 \%$ [6]. Some researchers have also reported that patients with preeclampsia also have an increased the risk for developing GDM [7]. Women with co-presence of these two diseases present a challenge for good clinical managment and have a significantly increased risk of an adverse pregnancy outcome.

The poor intrauterine environment caused by GDM and preeclampsia induces changes in fetal DNA methylation patterns related to fat synthesis, leading to abnormal NBW [8]. Dyslipidemia is an important risk factor affecting the growth and development of the fetus and may also be the critical pathogenesis of GDM 
with co-existent preeclampsia (GCP) $[9,10]$. Disturbances in lipid metabolism are a common cause of GDM and preeclampsia. Lipid levels are the result of the dynamic balance of synthesis and decomposition and are closely related to insulin sensitivity and insulin resistance [11]. Insulin resistance in pregnant women with GDM will lead to dyslipidemia, which damages the vascular function and further develops into preeclampsia.

To date, there has been a relative lack of research on the risk factors of abnormal NBW in women with GCP. In this study, we focused on the association between maternal glucose/lipid metabolism parameters and LGA or SGA.

\section{METHODS}

\section{Study Population and Eligibility Criteria}

All data used is this study were retrieved the electronic medical records (EMR) system of Women and Children's Hospital, Xiamen University (Xiamen, China), which is a tertiary care public hospital. All procedures performed in studies involving human participants were in accordance with the ethical standards ethics committee of Women and Children's Hospital, Xiamen University (ky-2019-006), and with the 1964 Helsinki Declaration and its later amendments or comparable ethical standards. Informed consent was obtained from all individual participants.

This study is a retrospective observational study. All personal identification information was replaced by a digital number to protect the privacy of the participants. All pregnant women who had received prenatal care and delivered their baby in Women and Children's Hospital, Xiamen University, between January 2016 and November 2018 were eligible to participate.

\section{Inclusion and Exclusion Criteria}

The inclusion criteria were: pregnant women with GCP who gave birth to a singleton without chromosomal abnormality and developmental malformation; both parents with no congenital defects or genetic diseases.

GDM diagnosis was made when glucose values exceeded the standard cutoff levels (fasting, $5.1 \mathrm{mmol} / \mathrm{L} ; 1 \mathrm{~h}, 10.0 \mathrm{mmol} / \mathrm{L}$; and $2 \mathrm{~h}, 8.50 \mathrm{mmol} / \mathrm{L}$ ) in a $75 \mathrm{~g}$ oral glucose tolerance test performed between 24 and 28 weeks of pregnancy [12]. Preeclampsia was diagnosed on the basis of systolic blood pressure (SBP) of $\geq 140 \mathrm{mmHg}$ ) and/or diastolic blood pressure (DBP) of $\geq 90 \mathrm{mmHg}$ after 20 weeks of gestation, proteinuria $\geq 0.3 \mathrm{~g} / 24 \mathrm{~h}$, or positive random urinary protein test, as well as upper abdominal discomfort, headache, and other known symptoms [13].

Exclusion criteria were: incomplete clinical data; drug abuse or alcohol abuse by the pregnant woman and her spouse; a smoking habit of the pregnant women; presence of hemoglobinopathy, tumors, mental disease, severe anemia, or massive blood loss during pregnancy.

\section{Data Collection and Maternal and Newborn Characteristics}

The researchers collected information on pregnant women and their newborns from the hospital's EMR system. The major characteristics assessed were LGA, SGA, severe preeclampsia (S-PE), and the maternal plasma glucose/ lipid profile in late pregnancy. The secondary characteristics assessed were maternal age, height, body mass index (BMI), gestational weight gain (GWG), abortion history, high education level (college diploma or above) or not, primipara or not, preterm or not, and fetal gender.

Abnormal NBW The newborns were classified as LGA when the NBW was $>90$ th percentile and as SGA when it was $<10$ th percentile, according to the gestational age and sexspecific INTERGROWTH-21st curves for China [14]. The remaining babies had a normal birth weight and were considered to be the control group.

Blood glucose control targets The blood glucose targets were a fasting plasma glucose $\leq 5.3 \mathrm{mmol} / \mathrm{L}$ and 1 -h postprandial glucose 
$<7.8 \mathrm{mmol} / \mathrm{L}$, as well as glycated hemoglobin $(\mathrm{HbA} 1 \mathrm{c}) \leq 6.5 \%$, according to American Diabetes Association (ADA) recommendation in 2020 [15]. High plasma glucose or high HbA1c refers to failure to achieve the above goals.

Hyperlipidemia Hyperlipidemia was defined as triglyceride $(\mathrm{TG}) \geq 2.3 \mathrm{mmol} / \mathrm{L}$, total cholesterol (TC) $\geq 6.2 \mathrm{mmol} / \mathrm{L}$, high-density lipoprotein cholesterol (HDL-C) $<1.0 \mathrm{mmol} /$ $\mathrm{L}$, and low-density lipoprotein cholesterol (LDL-C) $\geq 4.1 \mathrm{mmol} / \mathrm{L}$, according to the standards in China [16].

Preeclampsia severity S-PE is diagnosed by any of the following adverse conditions in patients with preeclampsia: continuous high blood pressure (SBP $\geq 160 \mathrm{mmHg}$ and/or DBP $\geq 110 \mathrm{mmHg}$ ); proteinuria $\geq 2.0 \mathrm{~g} / 24 \mathrm{~h}$ or random proteinuria $(++)$; serum creatinine $\geq 106 \mu \mathrm{m} / \mathrm{L} ; \quad$ platelet $<100,000 / \mathrm{mL}$; elevated blood LDH; elevated blood aspartate transaminase and alanine transaminase levels; persistent headache or other brain or visual impairments; and persistent upper abdominal pain [13].

Height and age classification Short figure was defined as height in the lowest quartile of adult women in China $(<155 \mathrm{~cm})$; high figure was defined as height in the highest quartile of adult women in China $(>161 \mathrm{~cm})$ [17]. Advanced age referred to $\geq 35$ years according to the the standard of pregnant women's risk assessment in China [18].

$B M I$ and GWG classification Prepregnancy BMI was calculated as weight (kg) divided by height $(\mathrm{m})$ squared. BMI categories were established according to the Working Group of Obesity in China (thin $<18.5$; normal 18.5-23.9; overweight/obese $>23.9$ [19]. The GWG is divided into three levels: insufficient, adequate, and excessive, according to the US Institute of Medicine (IOM) Guidelines 2009 [20]. In this study, we calculated four indicators: early insufficient or excessive GWG (before 28 weeks of gestation), and late insufficient or excessive GWG (after 28 weeks of gestation).

\section{Statistical Methods}

The SAS statistical package version 9.3 (SAS Institute Inc., Cary, NC, USA) was used for statistical analysis. Categorical variables were described by frequency. Comparisons among multiple group categorical variables were based on the Chi-square test for the $R \times C$ table. The Chi-square test for $2 \times 2$ table or the Fisher exact test was used for comparisons between categorical variables of two groups. The $p$ value was adjusted for multiple treatments. Logistic regression analysis was used to analyze the risk factors of SGA or LGA. The odds ratio (OR) and 95\% confidence intervals (CIs) for the association between glucose/lipid metabolism parameters of patients with GCP and abnormal NBW were determined. Factors for adjustment were maternal stature, prepregnancy BMI, GWG, among others. The significance level (alpha value) was set at $p<0.05$.

\section{RESULTS}

\section{Maternal and Newborn Clinical Characteristics in 248 Cases of GCP}

There was no difference in the ratio of advanced age, primipara, abortion history, and preterm delivery of the pregant women and the composition ratio of newborn sex (male fetus) between the control group and the LGA group or SGA group. The rate of short stature, prepregnancy thin, and early insufficient GWG was higher in the pregnant women in the SGA group than in those in the control group (30.3 vs. $13.8 \%, p<0.05 ; 24.2$ vs. $7.9 \%, p<0.05$; and 39.4 vs. $21.2 \%, p<0.05$, respectively). The proportion of maternal prepregnancy obesity was lower in the SGA group than in the control group ( 18.2 vs. $45.5 \%, p<0.05)$. The rate of high stature and early excessive GWG was higher in the pregnant women in the LGA group than in those in the control group (46.2 vs. $25.9 \%, p<0.05$ and 55.0 vs. $80.8 \%, p$ $<0.05$, respectively). There was no difference in the constituent ratio of late excessive GWG or late insufficient GWG between the control 
Table 1 Maternal and newborn clinical characteristics in the 248 cases of gestational diabetes mellitus and co-existent preeclampsia

\begin{tabular}{lllll}
\hline Character & SGA $(\boldsymbol{n}=\mathbf{3 3})$ & LGA $(\boldsymbol{n}=\mathbf{2 6})$ & Control $(\boldsymbol{n}=\mathbf{1 8 9})$ & $\boldsymbol{p}$ \\
\hline Advanced rate & $30.3 \%(10)$ & $19.2 \%(5)$ & $35.4 \%(67$ & 0.241 \\
Primipara rate & $72.7 \%(24)$ & $65.4 \%(17)$ & $59.8 \%(113)$ & 0.344 \\
Abortion history rate) & $42.4 \%(14)$ & $57.7 \%(15)$ & $48.1 \%(91)$ & 0.503 \\
High education rate & $75.8 \%(25)$ & $61.5 \%(16)$ & $68.3 \%(129)$ & 0.498 \\
Preterm rate & $21.2 \%(7)$ & $15.4 \%(4)$ & $18.0 \%(34)$ & 0.659 \\
Male fetal rate & $48.5 \%(16)$ & $57.7 \%(15)$ & $57.7 \%(109)$ & 0.612 \\
Short figure rate & $30.3 \%(10)^{*}$ & $7.7 \%(2)$ & $13.8 \%(26)$ & 0.027 \\
High figure rate & $18.2 \%(6)$ & $46.2 \%(12)^{*}$ & $25.9 \%(49)$ & 0.004 \\
Prepregnancy obesity rate & $18.2 \%(6)^{*}$ & $50.0 \%(15)$ & $45.5 \%(86)$ & 0.010 \\
Prepregnancy thin rate & $24.2(8)^{*}$ & $0.0 \%(0)$ & $7.9 \%(15)$ & 0.003 \\
Early excessive GWG rate & $42.4 \%(14)$ & $80.8 \%(21)^{*}$ & $5.0 \%(104)$ & 0.011 \\
Early insufficient GWG rate & $39.4 \%(13)^{*}$ & $3.8 \%(1) *$ & $21.2 \%(40)$ & 0.004 \\
Late excessive GWG rate & $45.5 \%(15)$ & $69.2(18)$ & $56.6 \%(10)$ & 0.187 \\
Late insufficient GWG rate & $30.3 \%(10)$ & $7.7 \%(2)$ & $25.9 \%(49)$ & 0.092 \\
\hline
\end{tabular}

Values in table are given as a percentage, with the number of newborns in that category given in parenthesis Control Normal newborn birth weight (NBW), $G W G$ gestational weight gain, $L G A$ large for gestational age (birth weight > 90th percentile), $S G A$ small for gestational age ( $<10$ th percentile)

*Statistically significant at $p<0.05$ compared with the control group

group and the LGA group or SGA group (Table 1).

\section{Maternal Glucose/Lipid Metabolism and Severity of Preeclampsia in 248 Cases of GCP}

Maternal glucose/lipid metabolism in the SGA group was associated with higher rates of S-PE compared to the control group (75.8 vs. $49.2 \%$, $p<0.05)$, while maternal glucose/lipid metabolism in the LGA group was associated with higher rates of high HbA1c (76.9 vs. $42.3 \%, p$ $<0.05$ ) and low HDL-C (73.1 vs. $44.4 \%, p$ $<0.05)$ compared to the control. There was no difference in the constituent ratios of high blood glucose, high TG, high TC, and high HDL-C between the control group and the LGA group or SGA group (Table 2).
We performed multivariate regression analysis of the association between the maternal glucose/lipid metabolism parameters and abnormal NBW in mothers with GCP (Table 3). Multivariate analysis of SGA revealed the following associations: S-PE (OR 3.226, 95\% CI 1.385-7.515; adjusted OR [AOR] 3.675, 95\% CI 1.467-9.207; $p<0.05)$; high HbA1c $(>6.5 \%)$ (OR 0.436, 95\% CI 0.187-1.017; AOR 0.459, 95\% CI 0.179-1.173; $p>0.05)$; low HDL-C $(<1.0 \mathrm{mmol} / \mathrm{L})(\mathrm{OR} 0.625,95 \%$ CI 0.287-1.361; AOR 0.637, 95\% CI 0.267-1.520; $p>0.05$ ). Multivariate analysis of LGA revealed the following associations: S-PE (OR 30.885, 95\% CI 0.398-2.013; AOR 0.974, 95\% CI 0.400-2.371; $p$ $>0.05$ ); high HbA1c (OR 4.542, 95\% CI 0.187-11.824; AOR 3.997, 95\% CI 1.452-10.998; $p<0.05$ ); low HDL (OR 3.393, 95\% CI 1.362-8.453; AOR 2.900, 95\% CI 1.100-7.647; $p<0.05$ ) (Table 3). 
Table 2 Maternal glucose/lipid metabolism and the severity of preeclampsia in 248 cases of gestational diabetes mellitus and co-existent preeclampsia

\begin{tabular}{lllll}
\hline Risk factor & SGA $(\boldsymbol{n}=\mathbf{3 3})$ & LGA $(\boldsymbol{n}=\mathbf{2 6})$ & Control $(\boldsymbol{n}=\mathbf{1 8 9})$ & $\boldsymbol{p}$ \\
\hline High HbAlc rate & $24.2 \%(8)$ & $76.9 \%(20)^{*}$ & $42.3 \%(80)$ & 0.000 \\
High plasma glucose rate & $15.2 \%(5)$ & $23.1 \%(6)$ & $31.2 \%(59)$ & 0.138 \\
S-PE & $75.8 \%(25)^{*}$ & $46.2 \%(12)$ & $49.2 \%(93)$ & 0.015 \\
High triglycerides rate & $15.2 \%(5)$ & $42.3 \%(11)$ & $25.4 \%(48)$ & 0.059 \\
High total cholesterol rate & $21.2 \%(7)$ & $15.4 \%(4)$ & $18.0 \%(34)$ & 0.841 \\
Low HDL rate & $33.3 \%(11)$ & $73.1 \%(19)^{*}$ & $44.4 \%(84)$ & 0.007 \\
High LDL rate & $18.2 \%(6)$ & $3.8 \%(1)$ & $13.8 \%(26)$ & 0.255 \\
\hline
\end{tabular}

Values in table are given as a percentage, with the number of newborns in that category given in parenthesis $H b A 1 c$ Glycated hemoglobin, $H D L$ high-density cholesterol, $L D L$ low-density cholesterol, $S-P E$

${ }^{*}$ Statistically significant at $p<0.05$ compared with the control group

Table 3 Multivariate regression analysis of the association between the maternal glucose/lipid metabolism parameters and the abnormal NBW in GCP

\begin{tabular}{lllll}
\hline Characteristics $^{\mathbf{a}}$ & Crude OR & $\mathbf{9 5 \%} \mathbf{C I}$ & Adjusted OR $^{\mathbf{b}}$ & 95\% CI \\
\hline S-PE for SGA & 3.226 & $1.385-7.515$ & 3.675 & $1.467-9.207^{*}$ \\
S-PE for LGA & 0.885 & $0.398-2.013$ & 0.974 & $0.400-2.371$ \\
High HbAlc for SGA & 0.436 & $0.187-1.017$ & 0.459 & $0.179-1.173$ \\
High HbAlc for LGA & 4.542 & $0.187-11.824$ & 3.997 & $1.452-10.998^{*}$ \\
Low HDL for SGA & 0.625 & $0.287-1.361$ & 0.637 & $0.267-1.520$ \\
Low HDL for LGA & 3.393 & $1.362-8.453$ & 2.900 & $1.100-7.647^{*}$ \\
\hline
\end{tabular}

$C I$ Confidence interval, $O R$ odds ratio

${ }^{*}$ Statistically significant compared with normal NBW group, $p<0.05$

a The reference value for all comparisons was that of the normal NBW

b The factors for adjustment were rate of short or high figure, prepregnancy obesity or thin, and early insufficient or excessive GWG of mothers

The factors for adjustment: maternal stature, prepregnancy BMI, GWG and others.

\section{DISCUSSION}

The exact pathogenesis of GCP is not clear, although compared with pregnant women without GCP, the dysfunction of glucose/lipid metabolism in GCP patients is more severe.

Continuous maternal hyperglycemia results in relatively high levels of glucose entering the fetal circulation through the placenta; in contrast, the insulin produced by the mother cannot pass through the placenta. Hyperglycemia can stimulate the proliferation of fetal islet $\beta$ cells and thereby the secretion of excessive insulin, increase the synthesis of protein and fat, and eventually lead to LGA [21]. The association of hyperglycemia and SGA needs to be confirmed in large-scale clinical studies. HbA1c is considered to be the most reliable biomarker for monitoring long-term blood glucose control, and its value can reflect the average blood 
glucose level in the past 2-3 months [22]. However, the HbA1c value can be affected by many factors, especially hemoglobin synthesis and iron loading. In the third trimester of pregnancy, these factors will change dramatically. Therefore, many researchers question its value as a suitable pregnancy biomarker [23]. However, it has also been reported that the high HbA1c of GDM pregnant women in late pregnancy is related to macrosomia [24]. The clinical analysis of GCP in this study shows that high HbA1c in late pregnancy is an independent risk factor for LGA, while high blood glucose in the same period is not associated with LGA. The reason for this may be that HbA1c reflects the average blood sugar over a relatively long period in the past, while blood glucose reflects the immediate blood glucose levels and is more likely to fluctuate.

The relationship between maternal lipid metabolism and NBW is very complex and controversial. Some researchers have reported that only in obese pregnant women is the HDL$C$ value in late pregnancy negatively correlated with NBW [25]. Mossayebi et al. reported that TG is a predictor of NBW in nondiabetic and nonobese pregnant women [26]. It has also been reported that the TC and LDL-C values in pregnant women are related to neonatal weight [27]. In our study, the low HDL-C value of pregnant women with GCP was associated with LGA, while other forms of dislipidemia were not related to abnormal NBW. Although the above findings suggest that the metabolic characteristics of lipids in pregnant women may affect fetal birth weight, the mechanism has not yet been elucidated. TG cannot be transported through the placenta, but it can be hydrolyzed to free fatty acids (FFA) by lipoprotein esterase in the placenta. A high level of FFA can result to high levels of fat and glucose being deposited in the fetus due to the passage of FFA through the placenta; this may ultimately lead to LGA infants [26]. The cholesterol of mothers can be transported to the fetus through the placenta and further regulate the synthesis of fetal cholesterol, thereby affecting the size and weight of the fetus [27].

Preeclampsia is a hypertension disease of pregnancy. Placental perfusion decreases in S-PE patients, resulting in acute atherosclerosis of placental blood vessels and decreased placental function, thereby hindering the transport of nutrients and oxygen and ultimately limiting fetal growth [28]. In our study, we found that SGA was positively correlated with the severity of preeclampsia in GCP.

There are a number of limitations to this study. The most important of these is that many risk factors affect NBW, and we included only a limited number of these factors in the study. Factors affecting the pregnant woman include presence of anemia or iron load, lifestyle, diet structure, and psychological state, and these may have an impact on the NBW. Regarding the father, factors such as height, weight, and other potential genetic factors may also be associated with birth weight. As this study is a retrospective analysis, the clinical information analyzed was based on existing data. Therefore, the analysis of many of the factors mentioned above was not included in this study due to the lack of records. Our aim is to carry out a multicenter prospective study in the future that will have an expanded sample size. This will enable us to conduct in-depth research by designing complete case report on patients.

There are some strengths to our study as well as future implications. Although there have been various studies on the factors affecting NBW, observations on pregnant women with GCP are infrequent. Once GCP has developed, the risk of adverse perinatal outcome is much higher than that in other pregnant women $[29,30]$. It is necessary to carry out an in-depth exploration of this patient population. Some researchers believe that the role of $\mathrm{HbA} 1 \mathrm{c}$ as a biomarker for monitoring pregnancy needs to be carefully evaluated. We found that HbA1c was an independent LGA risk factor in pregnant women with GCP. The relationship between maternal lipid profile and NBW has been the focus of many studies. We reporte here, for the first time, that low HDL-C value in GCP mothers in late pregnancy was an independent predictor of LGA; in contrast, values for other lipoproteins were not associated with abnormal birth weight. 


\section{CONCLUSIONS}

The critical indicators of GCP affecting the NBW are maternal HbA1c and HDL-C in the late trimester and the severity of preeclampsia. We also found that the height of the mother, prepregnancy BMI, and early GWG are related to the NBW. This study helps optimize the clinical intervention strategy for GCP.

\section{ACKNOWLEDGEMENTS}

We thank all participants of the study.

Funding. No funding or sponsorship was received for this study or publication of this article. The authors funded the Rapid Service Fee. All authors have full access to all of the data in this study and take full responsibility for the integrity of the data and accuracy of the data analysis.

Authorship. All named authors meet the International Committee of Medical Journal Editors (ICMJE) criteria for authorship for this article, take responsibility for the integrity of the work as a whole, and have given their approval for this version to be published.

Authorship Contributions. XZ performed the design of this study. YX collected data and wrote/edited the manuscript.

Disclosures. Xueqin Zhang and Yunshan Xiao have nothing to disclose.

Compliance with Ethics Guidelines. All procedures performed in studies involving human participants were in accordance with the ethical standards ethics committee of Women and Children's Hospital, Xiamen University (ky-2019-006), and with the 1964 Helsinki Declaration and its later amendments or comparable ethical standards. Informed consent was obtained from all individual participants.

Data Availability. The datasets generated and analyzed during the current study are available from the corresponding author on reasonable request.

Open Access. This article is licensed under a Creative Commons Attribution-NonCommercial 4.0 International License, which permits any non-commercial use, sharing, adaptation, distribution and reproduction in any medium or format, as long as you give appropriate credit to the original author(s) and the source, provide a link to the Creative Commons licence, and indicate if changes were made. The images or other third party material in this article are included in the article's Creative Commons licence, unless indicated otherwise in a credit line to the material. If material is not included in the article's Creative Commons licence and your intended use is not permitted by statutory regulation or exceeds the permitted use, you will need to obtain permission directly from the copyright holder. To view a copy of this licence, visit http:// creativecommons.org/licenses/by-nc/4.0/.

\section{REFERENCES}

1. Grantz KL, Hediger ML, Liu D, Buck Louis GM. Fetal growth standards: The NICHD fetal growth study approach in context with INTERGROWTH-21st and the World Health Organization Multicentre Growth Reference Study. Am J Obstet Gynecol. 2018;218[2 Suppl]:S641-S655.e28. https://doi.org/10.1016/j. ajog.2017.11.593.

2. Yadav S, Rastogi D. Small for gestational age: growth and puberty issues. Indian Pediatr. 2015;52(2):135-40.

3. Khambalia AZ, Algert CS, Bowen JR, Collie RJ, Roberts CL. Long-term outcomes for large for gestational age infants born at term. J Paediatr Child Health. 2017;53(9):876-81. https://doi.org/10. 1111/jpc. 13593.

4. American College of Obstetricians and Gynecologists. ACOG practice bulletin no. 202. Gestational hypertension and preeclampsia. Obstet Gynecol. 2019;133(1):e1-e25. https://doi.org/10.1097/aog. 0000000000003018

5. Zhu WW, Fan L, Yang HX, et al. Fasting plasma glucose at 24-28 weeks to screen for gestational 
diabetes mellitus: new environment from China. Diabetes Care. 2013;36:2038-40.

6. Sun Y, Sun WJ, Yang H. Risk factors for preeclampsia in pregnant Chinese women with abnormal glucose metabolism. Int J Gynecol Obstet. 2008;101(1):74-6.

7. Carr DB, Newton KM, Utzschneider KM, et al. Gestational diabetes or lesser degrees of glucose intolerance and risk of preeclampsia. Hypertens Pregnancy. 2011;30(2):153-63.

8. Perry VEA, Copping KJ, Miguel-Pacheco G, et al. The effects of developmental programming upon neonatal mortality. Clin North Am Food Anim Pract. 2019;35(2):289-302. https://doi.org/10.1016/ j.cvfa.2019.02.002.

9. Hajar SS, Abbasi RZ, Alizadeh F, et al. The relationship of hyperlipidemia with maternal and neonatal outcomes in pregnancy: a cross-sectional study. Int J Reprod Biomed (Yazd). 2019;17:739-48. https://doi.org/10.18502/ijrm.v17i10.5294.

10. Shen H, Liu X, Chen Y, et al. Associations of lipid levels during gestation with hypertensive disorders of pregnancy and gestational diabetes mellitus: a prospective longitudinal cohort study. BMJ Open. 2016;6(12):e013509. https://doi.org/10.1136/ bmjopen-2016-013509.

11. Tangvarasittichai S. Oxidative stress, insulin resistance, dyslipidemia and type 2 diabetes mellitus. World J Diabetes. 2015;6(3):456-80.

12. Wei Y, Yang H, Zhu W, et al. International Association of Diabetes and Pregnancy Study Group criteria is suitable for gestational diabetes mellitus diagnosis: further evidence from China. Chin Med J (Engl). 2014;127(20):3553-6.

13. Eiland E, Nzerue C, Faulkner M. Preeclampsia 2012. J Pregnancy. 2012;2012:586578. https://doi.org/10. $1155 / 2012 / 586578$.

14. Dai L, Deng C, Li Y, et al. Birth weight reference percentiles for Chinese. PLoS One. 2014;9(8): e104779. https://doi.org/10.1371/journal.pone. 0104779 .

15. American Diabetes Association. Standards of medical care in diabetes-2020 abridged for primary care providers. Clin Diabetes. 2020;38(1):10-38. https:// doi.org/10.2337/cd20-as01.

16. Joint Committee for the Prevention and Treatment of Dyslipidemia in Chinese Adults. Guidelines for the prevention and treatment of dyslipidemia in Chinese adults (2016 revised edition). Chin J Cardiol. 2016;44(10):833-50.
17. Lao TT, Hui ASY, Sahota DS, Leung TY. Maternal height and risk of hypertensive disorders in pregnancy. J Matern Fetal Neonatal Med. 2017;17:1-6. https://doi.org/10.1080/14767058.2017.1410786.

18. Shan D, Qiu PY, Wu YX, et al. Pregnancy outcomes in women of advanced maternal age: a retrospective cohort study from China. Sci Rep. 2018;8(1):12239. https://doi.org/10.1038/s41598-018-29889-3.

19. Zhou B; Cooperative Meta-analysis Group of the Working Group on Obesity in China. Predictive values of body mass index and waist circumference for risk factors of certain related diseases in Chinese adults-study on optimal cut-off points of body mass index and waist circumference in Chinese adults. Asia Pac J Clin Nutr. 2002;11[Suppl]: S685-93.

20. Institute of Medicine and National Research Council. Weight gain during pregnancy: reexamining the guidelines. Washington DC: The National Academies Press; 2009.

21. Kc K, Shakya S, Zhang H, et al. Gestational diabetes mellitus and macrosomia: a literature review. Ann Nutr Metab. 2015;66:14-20.

22. World Health Organization. Use of glycated hemoglobin (HbA1c) in the diagnosis of diabetes mellitus: abbreviated report of WHO consultation. Geneva: World Health Organization; 2011.

23. Zhang $X, X i a o ~ Y$, Fan $Y$. Investigating the reliability of HbA1c monitoring for blood glucose control during late pregnancy in patients with gestational diabetes mellitus (GDM) with and without $\beta$-thalassemia minor. Diabetes Ther. 2018;9(6):2305-13. https://doi.org/10.1007/s13300-018-0516-z.

24. Mendes N, Alves M, Andrade R, et al. Association between glycated albumin, fructosamine, and HbA1c with neonatal outcomes in a prospective cohort of women with gestational diabetes mellitus. Int J Gynaecol Obstet. 2019;146(3):326-32. https://doi.org/10.1002/ijgo.12897.

25. Misra VK, Trudeau S, Perni U, et al. Maternal serum lipids during pregnancy and infant birth weight: the influence of prepregnancy BMI. Obesity (Silver Spring). 2011;19:1476-81.

26. Mossayebi E, Arab Z, Rahmaniyan M, et al. Prediction of neonates' macrosomia with maternal lipid profile of healthy mothers. Pediatr Neonatol. 2014;55:28-34.

27. Wang $\mathrm{D}, \mathrm{Xu} \mathrm{S}$, Chen $\mathrm{H}$, et al. The associations between triglyceride to high-density lipoprotein cholesterol ratios and the risks of gestational diabetes mellitus and large-for-gestational-age infant. Clin Endocrinol. 2015;83:490-7. 
28. Carter EB, Conner SN, Cahill AG. Impact of fetal growth on pregnancy outcomes in women with severe preeclampsia. Pregnancy Hypertens. 2017;8: 21-5. https://doi.org/10.1016/j.preghy.2017.02. 002.

29. Zhang X, Xiao Y. The association between trimester-specific weight gain and severe preeclampsia/ adverse perinatal outcome in gestational diabetes mellitus complicated by preeclampsia: a retrospective case study. Diabetes Ther. 2019;10:725. https:// doi.org/10.1007/s13300-019-0589-3.

30. Oppermann MLDR, Alessi J, Hirakata VN, et al. Preeclampsia in women with pregestational diabetes-a cohort study. Hypertens Pregnancy. 2019;25:1-8. https://doi.org/10.1080/10641955. 2019.1704002. 